\title{
Editorial \\ The transition from ductal carcinoma in situ to invasive breast cancer: the other side of the coin
}

\author{
Stuart J Schnitt
}

Department of Pathology, Beth Israel Deaconess Medical Center, 330 Brookline Avenue, Boston, MA 02215, USA

Corresponding author: Stuart J Schnitt, sschnitt@bidmc.harvard.edu

Published: 27 February 2009

Breast Cancer Research 2009, 11:101 (doi:10.1186/bcr2228)

This article is online at http://breast-cancer-research.com/content/11/1/101

(c) 2009 BioMed Central Ltd

See related research article by Castro et al., http://breast-cancer-research.com/content/10/5/R87

\begin{abstract}
The factors associated with the progression of ductal carcinoma in situ (DCIS) to invasive breast cancer are poorly understood. Many studies of this subject focus on the role of molecular and genetic alterations in the neoplastic epithelial cells. However, emerging evidence suggests that transition from DCIS to invasive cancer is strongly dependent upon alterations in the microenvironment. The potential roles of myoepithelial cells and of stromal-epithelial interaction are of particular interest in this regard.
\end{abstract}

In a study published previously in Breast Cancer Research, Castro and colleagues [1] compared the gene expression signatures of epithelial cells isolated by laser capture microdissection (LCM) from non-neoplastic breast tissue, pure ductal carcinoma in situ (DCIS), DCIS associated with invasive breast cancer, and invasive breast cancer. They found that the expression signature of cells derived from DCIS associated with invasive breast cancer was very similar to that of cells of invasive carcinomas, but differed substantially from cells of pure DCIS.

The observation by Castro and colleagues that cells derived from DCIS associated with invasive cancers show many similarities at the level of gene expression with cells derived from invasive cancers [1] are consistent with the results of prior studies in which the expression signatures of DCIS and invasive cancers were compared. In those studies, very few genes were found to be differentially expressed in DCIS and invasive breast cancers, particularly those of similar grade, and these studies were unable to define signatures that distinguished between DCIS and invasive cancer $[2,3]$. The results of these prior studies suggest that the most dramatic changes in gene expression occur during the transition from normal epithelium to DCIS rather than in the transition from
DCIS to invasive breast cancer. The finding of Castro and colleagues that pure DCIS and DCIS associated with invasive cancer showed a substantial number of differentially expressed genes is of interest and appears to be at odds with this concept. However, these results must be interpreted with caution since only five cases of pure DCIS were studied. Furthermore, these five cases of pure DCIS consisted of a relatively homogenous group of primarily large lesions with high grade nuclei and HER2 overexpression that were compared to a more heterogenous group of DCIS associated with invasive cancer [1].

Castro and colleagues propose two possible explanations for the observation that neoplastic cells of DCIS show considerable molecular overlap with cells of invasive breast cancer [1]. The first possible explanation is that only a small number of genes is associated with the progression of DCIS to invasive breast cancer. The second explanation proposed is that the major molecular alterations associated with invasion are manifested before there is morphologic evidence of invasion at the level of the light microscope. Both of these explanations focus on alterations in the neoplastic epithelial cells as the key determinants of the transition from in situ to invasive breast cancer. An alternative, albeit not mutually exclusive, explanation is that the progression of DCIS to invasive breast cancer is strongly dependent upon microenvironmental factors, perhaps even more so than on genetic or molecular changes in the neoplastic epithelial cells themselves. In fact, there is now a growing body of evidence supporting a critical role for the tumor microenvironment in breast cancer progression even in its earliest, pre-invasive stages. Components of the microenvironment that have received particular attention in this regard are myoepithelial cells (MECs) and stromal cells (that is, fibroblasts and myofibroblasts).

$\mathrm{DCIS}=$ ductal carcinoma in situ; $\mathrm{LCM}=$ laser capture microdissection; $\mathrm{MEC}=$ myoepithelial cell. 
MECs surround mammary ducts and lobular acini and have important roles in normal mammary gland development and physiology [4,5]. In addition, MECs have natural tumor suppressor functions, including maintenance of the basement membrane, providing a physical barrier between epithelial cells and the surrounding stroma, and maintenance of epithelial cell polarity. Furthermore, experimental evidence has indicated that MECs produce factors that, through paracrine effects, inhibit tumor growth, invasion and angiogenesis [6].

While MECs are retained around ductal-lobular spaces containing DCIS, recent molecular studies have indicated that MECs that surround spaces involved by DCIS differ substantially from normal MECs in several respects [6-9]. When compared to normal MECs, DCIS-associated MECs show downregulation of a variety of genes involved in normal functions, including those for oxytocin receptor, laminin and thrombospondin, and upregulation of genes for chemokines that enhance epithelial cell proliferation, migration, invasion and stromal angiogenesis, such as SDF1/CXCL12 and CXCL 14. DCIS-associated MECs also show increased levels of enzymes involved in the degradation of extracellular matrix, such as matrix metalloproteinases [7]. In addition, a recent study utilizing methylation-specific digital karyotyping demonstrated distinct epigenetic changes in DCIS-associated MECs [9]. Further, the results of several studies have shown that DCIS-associated MECs show immunophenotypic differences from MECs in normal breast tissue. For example, Hilson and colleagues [10] found that expression of MEC markers such as smooth muscle myosin heavy chain, CD10 and cytokeratin 5/6 was reduced in the MECs surrounding DCIS in over $80 \%$ of the cases evaluated when compared with MECs in normal breast tissue. Taken together, these results provide strong evidence that, in many cases of DCIS, the associated MECs are abnormal. These findings raise the possibility that the progression of DCIS to invasive breast cancer may, at least in part, be due to MEC abnormalities that result in a loss of their normal tumor suppressor functions $[5,6]$.

Other factors in the microenvironment may also be important in regulating the transition from DCIS to invasive breast cancer, such as stromal-epithelial interactions. Stromal cells influence growth, differentiation, invasive behavior and polarity of normal breast epithelial cells and breast cancer cells in vitro and in vivo [11]. In addition, stromal alterations similar to those seen in invasive breast cancers are already evident in association with some cases of DCIS and even in some benign lesions such as radial scars. These include stromal angiogenesis, increased stromal cell expression of mRNAs for various extracellular matrix components, and increased stromal cell expression of proteases and cytokines [12-14].

The molecular determinants of the transition from in situ to invasive carcinoma in the breast remain to be more clearly elucidated. One approach to address this issue is that used in the study of Castro and colleagues, that is, to utilize LCM to isolate 'pure' populations of epithelial cells and to evaluate molecular alterations in these cells [1]. However, it should be noted that even when LCM is used, it is not possible to reliably separate epithelial cells from MECs and differences in gene expression between groups (particularly between nonneoplastic breast tissue and DCIS) could well reflect differences in expression of MEC-associated genes rather than epithelial cell-associated genes. Moreover, focusing on changes in the epithelial cells, while important, is tantamount to viewing only one side of a coin. Studies addressing this important question should not ignore the other side of the coin, that is, the potentially important role of the microenvironment in regulating the progression of DCIS to invasive breast cancer.

\section{Competing interests}

The author declares that they have no competing interests.

\section{References}

1. Castro NP, Osório CABT, Torres C, Bastos EP, Mourão-Neto M, Soares FA, Brentani HP, Carraro DM: Evidence that molecular changes in cells occur before morphological alterations during the progression of breast ductal carcinoma. Breast Cancer Res 2008, 10:R87.

2. Porter D, Lahti-Domenici J, Keshaviah A, Bae YK, Argani P, Marks J, Richardson A, Cooper A, Strausberg R, Riggins GJ, Schnitt S, Gabrielson E, Gelman R, Polyak K: Molecular markers in ductal carcinoma in situ of the breast. Mol Cancer Res 2003, 1:362-375.

3. Ma XJ, Salunga R, Tuggle JT, Gaudet J, Enright E, McQuary $P$, Payette T, Pistone M, Stecker K, Zhang BM, Zhou YX, Varnholt H, Smith B, Gadd M, Chatfield E, Kessler J, Baer TM, Erlander MG, Sgroi DC: Gene expression profiles of human breast cancer progression. Proc Natl Acad Sci USA 2003, 100:5974-5979.

4. Lakhani SR, O'Hare MJ: The mammary myoepithelial cell - Cinderella or ugly sister? Breast Cancer Res 2001, 3:1-4.

5. Barsky SH, Karlin NJ: Mechanisms of disease: breast tumor pathogenesis and the role of the myoepithelial cell. Nat Clin Pract Oncol 2006, 3:138-151.

6. Polyak K, Hu M: Do myoepithelial cells hold the key for breast tumor progression? J Mammary Gland Biol Neoplasia 2005, 10:231-247.

7. Allinen M, Beroukhim R, Cai L, Brennan C, Lahti-Domenici J, Huang H, Porter D, Hu M, Chin L, Richardson A, Schnitt S, Sellers WR, Polyak K: Molecular characterization of the tumor microenvironment in breast cancer. Cancer Cell 2004, 6:17-32.

8. Adriance MC, Inman JL, Petersen OW, Bissell MJ: Myoepithelial cells: good fences make good neighbors. Breast Cancer Res 2005, 7:190-197.

9. Hu M, Yao J, Cai L, Bachman KE, van den Brûle F, Velculescu V, Polyak K: Distinct epigenetic changes in the stromal cells of breast cancers. Nat Genet 2005, 37:899-905.

10. Hilson JB, Schnitt SJ, Collins LC: Phenotypic alterations in ductal carcinoma in situ-associated myoepithelial cells: biological and diagnostic implications. Am J Surg Pathol 2009, 33:227-232.

11. Kim JB, Stein R, O'Hare MJ: Tumour-stromal interactions in breast cancer: the role of stroma in tumourigenesis. Tumour Biol 2005, 26:173-185.

12. Guidi AJ, Fischer L, Harris JR, Schnitt SJ: Microvessel density and distribution in ductal carcinoma in situ of the breast. J Natl Cancer Inst 1994, 86:614-619.

13. Brown LF, Guidi AJ, Schnitt SJ, Van De Water L, Iruela-Arispe ML, Yeo TK, Tognazzi K, Dvorak HF: Vascular stroma formation in carcinoma in situ, invasive carcinoma, and metastatic carcinoma of the breast. Clin Cancer Res 1999, 5:1041-1056.

14. Kleer CG, Bloushtain-Qimron N, Chen $\mathrm{YH}$, Carrasco D, Hu M, Yao J, Kraeft SK, Collins LC, Sabel MS, Argani P, Gelman R, Schnitt SJ, Krop IE, Polyak K: Epithelial and stromal cathepsin $\mathrm{K}$ and CXCL14 expression in breast tumor progression. Clin Cancer Res 2008, 14:5357-5367. 